\title{
Coronariografia Via Transradial: Curva de Aprendizagem, Avaliada por Estudo Multicêntrico
}

\author{
André Labrunie ${ }^{1,2,3}$, Marden Andre Tebet ${ }^{1,2}$, Pedro Beraldo de Andrade ${ }^{1,2}$, \\ Mônica Vieira Athanazio de Andrade ${ }^{1}$, Lucieni de Oliveira Conterno ${ }^{1,4}$, Luiz Alberto Mattos ${ }^{1,5}$, \\ Fábio Cardoso de Carvalho ${ }^{3}$, Edson Antonio Bregagnollo ${ }^{3}$
}

\section{RESUMO}

Introdução: A via de acesso femoral tem sido a preferida para os procedimentos diagnósticos e terapêuticos coronarianos, mas apresenta limitações, principalmente relacionadas a complicações vasculares e hemorrágicas. O acesso transradial é uma técnica mais recentemente empregada com o objetivo principal de diminuir essas complicações, além de produzir maior conforto e redução dos custos de hospitalização, embora com maior curva de aprendizagem. O objetivo deste estudo foi avaliar a realização de coronariografia transradial, executada por operadores sem experiência na técnica, e comparar com a abordagem clássica (Sones e femoral) em termos de sucesso do procedimento e complicações, analisando a influência da curva de aprendizagem. Método: Estudo multicêntrico realizado em 14 hospitais do interior de São Paulo, no período de um ano. Foram randomizados mil pacientes de maneira equivalente para as técnicas transradial ou clássica. Resultados: A taxa de sucesso em ambos os grupos foi similar $(97,8 \%$ vs. 98,5\%; $\mathrm{P}=0,47)$. No grupo clássico, $95,2 \%$ dos procedimentos foram realizados pela técnica de Sones. O número de cateteres utilizados, a duração do procedimento e o tempo de exposição aos raios $X$ foram maiores no grupo transradial $(P<0,001)$. Não houve diferença quanto às complicações maiores (morte, infarto e acidente vascular cerebral) e quanto às complicações vasculares e hemorrágicas. Os grupos que realizaram mais de 100 procedimentos pela artéria radial (3 centros/5 operadores) obtiveram menor taxa de insucesso $(1,6 \%$ vs. $3,6 \% ; P=0,04)$. Conclusão: Um período de aprendizagem para a realização de procedimentos pela via transradial mostra-se necessário, porém não se acompanha de menor índice de sucesso, nem tampouco de maior taxa de complicações para os pacientes.

DESCRITORES: Artéria radial. Angiografia coronária. Aprendizagem.

\footnotetext{
1 Irmandade da Santa Casa de Misericórdia de Marília - São Paulo, SP, Brasil.

2 Hospital do Coração de Londrina - Londrina, PR, Brasil.

3 Hospital das Clínicas - Faculdade de Medicina/UNESP (Botucatu)

- Botucatu, SP, Brasil.

${ }^{4}$ Faculdade Estadual de Medicina de Marília - Marília, SP, Brasil.

${ }^{5}$ Instituto Dante Pazzanese de Cardiologia - São Paulo, SP, Brasil.

Correspondência: Marden Andre Tebet. Avenida Carlos Artencio,

356/43B - Fragata - Marília, SP, Brasil - CEP 17519-255

E-mail: mardentebet@gmail.com

Recebido em: 30/10/2008 • Aceito em: 27/2/2009
}

\section{ABSTRACT}

Transradial Coronary Angiography: Learning Curve, Evaluated by a Multicenter Study

Background: Femoral access for diagnostic and therapeutic coronary procedures has been the dominant approach worldwide, despite an increased risk of vascular and hemorrhagic complications. The transradial approach is a more recent technique used to reduce these complications, providing more comfort and reducing hospitalization costs. However, it is associated with an inherent learning curve. The aim of the study was to evaluate transradial coronary angiography performed by inexperienced operators and compare the success and complication rates of this technique with the classical approach (Sones and femoral), analyzing the influence of the learning curve. Methods: Multicenter, randomized study, in 14 hospitals in São Paulo State in a one-year period. One thousand patients were randomized to the transradial or classical techniques. Results: The success rate was similar in both groups $(97.8 \%$ vs. $98.5 \%$; $P=0.47)$. Sones technique was used in $95.2 \%$ of the procedures in the classical technique group. The number of catheters used, the duration of the procedure and X-ray exposure were greater in the transradial group $(P<0.001)$. There were no differences in the major cardiac adverse events (death, infarct and stroke), and vascular and bleeding complications. Operators who had performed over 100 procedures using the transradial approach (3 centers $/ 5$ operators) had a lower failure rate $(1.6 \%$ vs. $3.6 \%$; $\mathrm{P}=0.04)$. Conclusion: A learning period to perform coronary procedures using the transradial approach is required, but it is not associated with an increased risk of procedure failure or vascular complications compared with Sones or femoral approaches.

DESCRIPTORS: Radial artery. Coronary angiography. Learning. 
A coronariografia é o exame de escolha para o diagnóstico de aterosclerose coronariana obstrutiva e foi originalmente realizada pela arteriotomia braquial aberta (técnica de Sones). Posteriormente, o desenvolvimento de procedimentos por punção arterial percutânea permitiu um acesso mais rápido e fácil à circulação coronariana.

Desde então, a artéria femoral tem sido o sítio de acesso preferido para os procedimentos coronarianos (técnica de Judkins), mas apresenta algumas limitações. As complicações vasculares e hemorrágicas que ocorrem no sítio de punção podem ser significantes em termos de morbidade, e são mais prováveis de ocorrer quando o procedimento é feito sob um regime agressivo de anticoagulação e antiagregação'

A abordagem transradial para a coronariografia foi introduzida por Campeau², em 1989. A localização superficial da artéria radial permite fácil compressão, com subsequente diminuição das complicações hemorrágicas. Além do mais, nenhum nervo ou veia expressivos estão no trajeto anatômico do sítio de punção, minimizando os riscos de injúria ${ }^{3}$. A deambulação precoce, permitida pelo acesso radial, produz maior conforto e reduz os custos da hospitalização ${ }^{4-6}$. No entanto, o acesso transradial é algumas vezes tecnicamente mais trabalhoso e delicado, demandando maior período de aprendizagem para os operadores ${ }^{7-9}$.

O objetivo do presente estudo foi avaliar se o período de aprendizagem da técnica transradial, realizada por operadores treinados nas técnicas braquial e femoral, influencia a incidência de complicações relacionadas ao procedimento.

\section{MÉTODO}

O estudo foi conduzido em 14 centros participantes no Brasil ${ }^{10}$. No período de um ano foram randomizados 1.000 pacientes para o grupo transradial $(\mathrm{n}=502)$ ou para o grupo clássico $(\mathrm{n}=498)$. A randomização foi feita por meio de envelopes selados.

Considerando a curva de aprendizagem, os 14 centros participantes foram divididos em dois grupos: grupo A, com centros que fizeram mais de 100 coronariografias transradiais; e grupo B, com aqueles que fizeram menos de 100 procedimentos. Todos os médicos participantes eram largamente experientes em coronariografia pelos acessos femoral e braquial.

A indicação de coronariografia foi feita pelo cardiologista clínico, mediante sintomas ou alterações de provas funcionais que sugerissem doença arterial coronariana.

Como critério de inclusão, era necessário teste de Allen normal para verificar a adequada fonte de circulação colateral pela artéria ulnar. O teste consistia no fechamento e na abertura da mão com compressão simultânea das artérias radial e ulnar. No teste normal, a mão retorna à coloração habitual dentro de 10 segundos depois da descompressão da artéria ulnar, enquanto a artéria radial permanece comprimida.

Os critérios de exclusão foram: cirurgia de revascularização prévia, doença vascular conhecida nos membros superiores, cateterização direita e a possibilidade de uso de dispositivos, como marcapasso temporário ou balão intra-aórtico, durante o procedimento. Idade, sexo ou peso corporal não foram limitações para a inclusão no estudo.

O protocolo foi aprovado pelo Comitê de Ética em Pesquisa de cada instituição participante, tendo sido obtido termo de consentimento esclarecido de todos os pacientes incluídos no estudo.

O procedimento transradial foi realizado por meio de punção arterial após anestesia local (1-3 ml de lidocaína a $2 \%$ ), utilizando-se agulha com cateter de polietileno tipo Jelco ${ }^{\mathrm{TM}}$ calibre 20. Posteriormente era inserido um guia metálico (reto, com $30 \mathrm{~cm}$ de extensão). Previamente à passagem do fio-guia, todos os pacientes receberam heparina (5.000 UI) e mononitrato de isossorbida $(10 \mathrm{mg}$ ) através do cateter intra-arterial. Um introdutor $5 \mathrm{Fr}$ foi posicionado sobre o fio-guia e um cateter multipropósito tipo MPA $5 \mathrm{Fr}$ foi utilizado para a angiografia. A ventriculografia esquerda foi primeiramente realizada, seguida da cateterização seletiva das artérias coronárias direita e esquerda. Quando não havia sucesso com a utilização do cateter multipropósito, outros foram usados (Judkins, Amplatz, pig-tail). Imediatamente após o procedimento, o introdutor era removido, sendo obtida hemostasia com uma bandagem compressiva aplicada por 1-2 horas.

A abordagem clássica foi realizada utilizando-se arteriotomia braquial (técnica de Sones) ou punção da artéria femoral percutânea (técnica de Judkins).

A hemostasia foi obtida com sutura arterial ou compressão manual para os procedimentos realizados pela braquial ou femoral, respectivamente.

A qualidade angiográfica foi avaliada pelo operador em uma escala de 0 a 3: 0 = angiografia não realizada; 1 = vasos não suficientemente contrastados para correto diagnóstico; 2 = vasos suficientemente contrastados; 3 = vasos idealmente contrastados. A dificuldade em realizar o procedimento foi analisada por outra escala: $0=$ nenhuma; 1 = pouca; 2 = intermediária; 3 = extrema. A ocorrência ou não de espasmo da artéria radial foi classificada em: 0 = espasmo ausente; 1 = espasmo leve; 2 = espasmo moderado; 3 = espasmo importante.

A análise estatística foi realizada usando o programa estatístico SPSS para Windows 6.1. As variáveis contínuas foram expressas em médias e desvio padrão e comparadas com o teste $t$ de Student. As variáveis categóricas foram expressas em números absolutos ou 
relativos e comparadas utilizando-se o teste de quiquadrado. Análise de regressão logística foi realizada para identificar as características basais associadas com o sucesso do procedimento e as complicações. Considerou-se estatisticamente significante $\mathrm{P}<0,05$.

\section{RESULTADOS}

As características clínicas basais são apresentadas na Tabela 1. Ambos os grupos eram similares quanto a sexo, diabetes melito e indicação clínica para a coronariografia. Houve predomínio de pacientes mais jovens $(57,2 \pm 13,1 \%$ vs. $59,1 \pm 11 \% ; P<0,006)$, hipertensão arterial (48,6\% vs. 42,1\%; $\mathrm{P}<0,004)$, tabagismo $(31,4 \%$ vs. 23,1\%; P $<0,003)$ e índice de massa corporal $>25 \mathrm{~kg} / \mathrm{m}^{2}(68,3 \%$ vs. 57,4\%; P < 0,001) no grupo transradial. Por outro lado, houve maior frequência de história familiar de doença arterial coronariana $(27,1 \%$ vs. $35,3 \%$; $\mathrm{P}<0,005)$ no grupo clássico.

A taxa de sucesso em ambos os grupos foi similar (97,8\% vs. 98,5\%; $P=0,47)$ (Tabela 2). Não se obteve sucesso em $11(2,2 \%)$ casos do grupo transradial. Desses, $8(1,6 \%)$ foram por espasmo, sendo 4 na punção arterial, com desaparecimento do pulso, e os outros 4 casos após a punção, com demonstração angiográfica de espasmo no trajeto arterial. Os outros 3 casos de insucesso foram decorrentes de extrema tortuosidade vascular. No grupo clássico, 474 (95,2\%) procedimentos foram realizados via artéria braquial e $24(4,8 \%)$, pela punção da artéria femoral.

A Tabela 2 resume os achados angiográficos. O número de cateteres utilizados em cada grupo foi significantemente maior no grupo transradial $(1,43 \pm$ $0,70$ vs. $1,25 \pm 0,75 ; P<0,001)$. Apenas um cateter foi utilizado em 346 pacientes no grupo transradial e em 405 pacientes no grupo clássico (68,9\% vs. 81,3\%; $P<0,001)$.

A duração do procedimento e o tempo de exposição aos raios $X$, um confiável marcador de complexidade do procedimento, foi, em média, 2,7 e 1,9 minutos maior no grupo transradial, respectivamente, em comparação com o grupo clássico $(P<0,001)$.

Os espasmos predominaram no grupo transradial, sejam eles de graus leve $(10,7 \%$ vs. $3,4 \%$; $\mathrm{P}<0,01)$, moderado $(4,4 \%$ vs. $1,8 \% ; \mathrm{P}<0,01)$ ou grave $(5,7 \%$ vs. $0,8 \% ; P<0,001)$. A ocorrência de espasmos não inviabilizou a realização do procedimento.

TABELA 1

Características clínicas basais

\begin{tabular}{lccc}
\hline & Grupo transradial $(\mathbf{n}=\mathbf{5 0 2})$ & Grupo clássico $(\mathbf{n}=\mathbf{4 9 8})$ & P \\
\hline Idade (anos \pm DP) & $57,2 \pm 13,1$ & $59,1 \pm 11$ & $<0,006$ \\
Sexo masculino, $\mathrm{n}(\%)$ & $358(71,3)$ & $352(70,6)$ & 0,83 \\
Diabetes melito, $\mathrm{n}(\%)$ & $65(12,9)$ & $56(11,0)$ & 0,43 \\
História familiar de DAC, $\mathrm{n}(\%)$ & $136(27,1)$ & $176(35,3)$ & $<0,005$ \\
Hipertensão arterial, $\mathrm{n}(\%)$ & $244(48,6)$ & $210(42,1)$ & $<0,004$ \\
Tabagismo, $\mathrm{n}(\%)$ & $151(31,4)$ & $115(23,1)$ & $<0,003$ \\
$\mathrm{IMC}>25 \mathrm{~kg} / \mathrm{m}^{2}, \mathrm{n}(\%)$ & $343(68,3)$ & $286(57,4)$ & $<0,001$
\end{tabular}

$\mathrm{DAC}$ = doença arterial coronariana; $\mathrm{DP}=$ desvio padrão; $\mathrm{IMC}$ = índice de massa corporal (peso/altura²); $\mathrm{n}=$ número de pacientes.

TABELA 2

Resultados angiográficos

\begin{tabular}{lccc}
\hline & Grupo transradial $(\mathbf{n}=\mathbf{5 0 2})$ & Grupo clássico $(\mathbf{n}=\mathbf{4 9 8})$ & P \\
\hline Sucesso do procedimento (\%) & 97,8 & 98,5 & 0,47 \\
Complicações (\%) & 4,4 & 3,6 & 0,62 \\
Tempo de raios X (min) & $6,0 \pm 4,6$ & $4,1 \pm 2,7$ & $<0,001$ \\
Duração do procedimento (min) & $22,2 \pm 11,6$ & $19,5 \pm 10,3$ & $<0,001$ \\
Número de cateteres (n + DP) & $1,43 \pm 0,70$ & $1,25 \pm 0,75$ & $<0,001$ \\
Qualidade angiográfica & $2,29 \pm 1,22$ & $2,73 \pm 0,83$ & $<0,001$ \\
Dificuldade do procedimento & $0,28 \pm 0,68$ & $0,19 \pm 0,29$ & $<0,001$ \\
\hline
\end{tabular}

DP = desvio padrão; $\mathrm{n}=$ número de pacientes. 
Não houve diferenças entre os grupos quanto às complicações, tendo sido registrado um óbito em cada grupo $(\mathrm{P}>0,99)$. As complicações vasculares e hemorrágicas ocorreram em $9(1,7 \%)$ pacientes do grupo transradial e em $7(1,4 \%)$ do grupo clássico $(P=0,80)$. Oclusão arterial ocorreu em 12 pacientes do grupo transradial e em 10 pacientes do grupo clássico (2,4\% vs. $2 \% ; P=0,83)$, sendo todos esses casos assintomáticos.

Uma análise de regressão logística mostrou que nenhuma das características basais pode predizer o sucesso do procedimento ou a ocorrência de complicações.

A qualidade angiográfica foi significantemente meIhor no grupo clássico $(2,29 \pm 1,22$ vs. 2,73 $\pm 0,83$; $P<0,001)$.

Em relação à curva de aprendizagem, o grupo $\mathrm{A}$, com 3 centros e 5 operadores, realizou $749(74,9 \%)$ procedimentos e o grupo B, com 11 centros e 14 operadores, realizou $251(25,1 \%)$. Comparando-se os grupos, observou-se menor taxa de insucesso no grupo $\mathrm{A}(1,6 \%$ vs. $3,6 \%$; $\mathrm{P}=0,04)$. Embora maior número de complicações tenha sido observado no grupo B $(2,2 \%$ vs. $1,6 \%)$, não foi encontrada diferença estatística entre os grupos $(P=0,64)$.

\section{DISCUSSÃO}

O acesso transradial é uma alternativa atrativa para a realização da angiografia e da intervenção coronariana ${ }^{11,12}$, com algumas vantagens comparando-se à abordagem tradicional $\left.\right|^{13}$. A artéria radial, diferente das artérias femoral e braquial, não é uma artéria terminal; logo, quando o teste de Allen é normal, sua oclusão não compromete a perfusão da mão. $O$ curso superficial da artéria radial distal e sua proximidade com o osso favorecem a compressão, permitindo aos pacientes rápida movimentação após a retirada do introdutor, melhorando o conforto do paciente. Indivíduos que se submeteram a procedimentos invasivos coronarianos pelas vias clássica ou radial expressam nítida preferência pela última abordagem ${ }^{5}$. Ademais, a possibilidade da realização de intervenção coronariana percutânea ambulatorial pode reduzir os custos e a extensão do período de hospitalização ${ }^{14-16}$.

Essa abordagem, mesmo durante a aprendizagem, é altamente segura quando realizada por operadores experientes nas técnicas clássicas, produzindo uma similar taxa de complicações ${ }^{17,18}$. De fato, essa evidência é clara em todos os estudos em que o período de aprendizagem foi essencial, embora não haja consenso na literatura quanto ao número de procedimentos necessários para definir essa curva. Cooper et al. ${ }^{5}$ consideraram o número mínimo de 50 casos para classificar o intervencionista como experiente para realização de coronariografias por via transradial. Spaulding et al. ${ }^{19} \mathrm{e}$ Ludman et al. ${ }^{20}$ estabeleceram 20 procedimentos como o limite da inexperiência e Hildick-Smith et al. ${ }^{21}$ situam o intervalo de 20 a 40 casos como sendo necessários para a curva de aprendizagem. Esse estudo mostra a relevância do período de aprendizagem, verificando-se que ocorre maior taxa de sucesso no grupo que fez mais de 100 procedimentos. Quanto à duração do procedimento e da exposição radiológica, a abordagem transradial foi mais demorada que a abordagem clássica nesse estudo, fato não observado em outros artigos.

A necessidade de maior número de cateteres usados no grupo transradial é também um sinal indireto de maior complexidade da técnica. Adicionalmente, a análise de uma escala subjetiva do grau de dificuldade nos leva na mesma direção. Isso se explica por ser esse um estudo de curva de aprendizagem, ou seja, a falta de experiência do operador no acesso transradial tem papel maior na extensão do procedimento e no tempo de exposição aos raios $X^{22}$. Preocupações quanto ao aumento da exposição à radiação nos operadores primários podem ser diminuídas pelo posicionamento do braço junto do paciente e pelo uso de campos de proteção de chumbo com movimentação livre e suporte superior. A exposição do operador à radiação não foi avaliada nesse estudo. $\mathrm{O}$ uso de um cateter mutipropósito mais efetivo, especialmente desenhado para a abordagem radial, poderia proporcionar significativa vantagem, reduzindo o tempo do procedimento e a exposição aos raios $X$.

As complicações isquêmicas relatadas são muito raras, sendo a mais frequente a trombose da artéria radial, precoce e tardia, em geral assintomática, variando de $3 \%$ a $8 \%{ }^{23,24}$. O índice de recanalização tardia dessas artérias chega a 50\% ${ }^{25}$. Múltiplos fatores podem contribuir para a oclusão arterial, sendo o mais importante, sem dúvida, o tempo de canulação do vaso, pois quanto maior também maior a incidência de trombose; os outros preditores são o calibre do introdutor utilizado, a idade do paciente, assim como a dose de heparina empregada ${ }^{23}$.

Hematomas de grande volume são raros e potencialmente perigosos, podendo levar a síndrome compartimental com grande distensão de tecidos do antebraço, comprimindo as artérias radial e ulnar de forma simultânea, com consequente isquemia da mão e edema por comprometimento do retorno venoso, embora extremamente rara. Tizón-Marcos e Barbeau ${ }^{26}$ avaliaram recentemente a incidência de síndrome compartimental em 51.296 procedimentos realizados numa mesma instituição e encontram taxa de 0,004\%.

Este é um estudo multicêntrico, que, mais que uma comparação de operadores, é uma comparação de técnicas que representam a prática no Brasil. Embora tenha sido um estudo randomizado, houve algumas diferenças nas características basais entre os grupos; porém, a análise de regressão logística demonstrou que nenhuma das características clínicas avaliadas pôde predizer o resultado do procedimento. 
O acesso transradial já é popular na Europa e na Ásia, mas tem baixa aceitação na América, sendo o período de aprendizagem apontada como um dos maiores impedimentos para sua adoção.

\section{CONCLUSÃO}

O período de aprendizagem para utilização do acesso transradial para realização de coronariografia envolve maior dificuldade técnica, representada por maior número de cateteres necessários para a conclusão do procedimento, maior tempo total de procedimento e de exposição radiológica, e maior ocorrência de espasmo arterial. Entretanto, essa aprendizagem, necessária para a incorporação de uma nova técnica que traz consigo indiscutíveis vantagens, não se acompanhou de menor índice de sucesso, nem de maior taxa de complicações para os pacientes.

\section{AGRADECIMENTOS}

Agradecemos aos drs. Roberto V. Botelho e Samir A. Reis, pelo treinamento ministrado aos operadores e pela tabulação dos resultados, respectivamente.

\section{CONFLITO DE INTERESSES}

Os autores declararam inexistência de conflito de interesses.

\section{REFERÊNCIAS BIBLIOGRÁFICAS}

1. Jolly SS, Amlani S, Hamon M, Yusuf S, Mehta SR. Radial versus femoral access for coronary angiography or intervention and the impact on major bleeding and ischemic events: a systematic review and meta-analysis of randomized trials. Am Heart J. 2009;157(1):132-40.

2. Campeau L. Percutaneous radial artery approach for coronary angiography. Cathet Cardiovasc Diagn. 1989;16(1):3-7.

3. Hildick-Smith DJ, Lowe MD, Walsh JT, Ludman PF, Stephens NG, Schofield PM, et al. Coronary angiography from the radial artery: experience, complications and limitations. Int J Cardiol. 1998;64(3):231-9.

4. Mann JT 3 $3^{\text {rd }}$, Cubeddu MG, Schneider JE, Arrowood M. Right radial access for PTCA: a prospective study demonstrates reduced complications and hospital changes. J Invasive Cardiol. 1996;8(Suppl D):D40-4.

5. Cooper CJ, El-Shiekh RA, Cohen DJ, Blaesing L, Burket MW, Basu A, et al. Effect of transradial access on quality of life and cost of cardiac catheterization: a randomized comparison. Am Heart J. 1999;138(3 Pt 1):430-6.

6. Slagboon T, Kiemeneij F, Laarman GJ, van der Wieken R, Odekerken D. Actual outpatient PTCA: results of the OUTCLAS pilot study. Catheter Cardiovasc Interv. 2001;53(2):204-8.

7. Louvard Y, Pezzano M, Scheers L, Koukoui F, Marien C, Benaim $\mathrm{R}$, et al. Coronary angiography by a radial artery approach: feasibility, learning curve. One operator's experience. Arch Mal Coeur Vaiss. 1998;91(2):209-15.

8. Goldberg SL, Renslo R, Sinow R, French WJ. Learning curve in the use of the radial artery as vascular access in the performance of percutaneous transluminal coronary angioplasty. Cathet Cardiovasc Diagn. 1998;44(2):147-52.

9. Nunes GL, Oliveira AT, Alves L, Alfonso T. Influência da curva de aprendizado no sucesso e na ocorrência de complica- ções associadas aos procedimentos pela via radial. Rev Bras Cardiol Invas. 2007;15(2):115-8.

10. Labrunie A. Coronariografia por técnica transradial: a curva de aprendizagem, avaliada por estudo multicêntrico, randomizado, comparativo [Tese de doutorado]. Botucatu: UNESP - Faculdade de Medicina; 2003. p.149.

11. Bagger $\mathrm{H}$, Kristensen JH, Christensen PD, Klausen IC. Routine transradial coronary angiography in unselected patients. J Invasive Cardiol. 2005;17(3):139-41.

12. Kiemeneij F, Laarman GJ, Odekerken D, Slagboom T, van der Wieken R. A randomized comparison of percutaneous transluminal coronary angioplasty by the radial, brachial and femoral approaches: the access study. J Am Coll Cardiol. 1997;29(6):1269-75.

13. Louvard $Y$, Lefèvre T, Allain A, Morice M. Coronary angiography through the radial or the femoral approach: the CARAFE study. Cathether Cardiovasc Interv. 2001;52(2):181-7.

14. Lundén $M H$, Bengtson $A$, Lundgren SM. Hours during and after coronary intervention and angiography. Clin Nurs Res. 2006;15(4):274-89.

15. Bertrand OF, De Larochellière R, Rodés-Cabau J, Proulx G, Gleeton O, Nguyen CM, et al. A randomized study comparing same-day home discharge and abciximab bolus only to overnight hospitalization and abciximab bolus and infusion after transradial coronary stent implantation. Early Discharge After Transradial Stenting of Coronary Arteries Study Investigators. Circulation. 2006;114(24):2636-43.

16. Wiper A, Kumar S, MacDonald J, Roberts DH. Day case transradial coronary angioplasty: a four-year single-center experience. Catheter Cardiovasc Interv. 2006;68(4):549-53.

17. Archbold RA, Robinson NM, Schilling RJ. Radial artery access for coronary angiography and percutaneous coronary intervention. BMJ. 2004;329(7463):443-6.

18. Agostoni P, Biondi-Zoccai GG, Benedictis $\mathrm{ML}$, Rigattieri $\mathrm{S}$, Turri $M$, Anselmi $M$, et al. Radial versus femoral approach for percutaneous coronary diagnostic and interventional procedures: systematic overview and meta-analysis of randomized trials. J Am Coll Cardiol. 2004;44(2):349-56.

19. Spaulding $C$, Lefèvre T, Funck F, Thébault B, Chauveau $M$, Ben Hamda K, et al. Left radial approach for coronary angiography: results of a prospective study. Cathet Cardiovasc Diagn. 1996;39(4):365-70.

20. Ludman PF, Stephens NG, Harcombe A, Lowe MD, Shapiro LM, Schofield PM, et al. Radial versus femoral approach for diagnostic coronary angiography in stable angina pectoris. Am J Cardiol. 1997;79(9):1239-41.

21. Hildick-Smith DJ, Ludman PF, Lowe MD, Stephens NG, Harcombe AA, Walsh JT, et al. Comparison of radial versus brachial approaches for diagnostic coronary angiography when the femoral approach is contraindicated. Am J Cardiol. 1998;81(6):770-2.

22. Lange HW, von Boetticher $H$. Randomized comparison of operator radiation exposure during coronary angiography and intervention by radial or femoral approach. Catheter Cardiovasc Interv. 2006;67(1):12-6.

23. Barbeau GR, Gleeton O, Gobeil JF. Predictors of failure of transradial approach for coronary angiography and interventions: a multivariate analysis of a large series. Circulation. 1999;100(Suppl I):1603.

24. Barbeau GR, Gleeton O, Juneau C. Transradial approach for coronary angiography and interventions: procedural results and vascular complications from a series of 7049 procedures. Circulation. 1999;100(Supp| I):1598.

25. Bedford RF. Long-term radial artery cannulation: effects on subsequent vessel function. Crit Care Med. 1978;6(1):64-7.

26. Tizón-Marcos H, Barbeau GR. Incidence of compartment syndrome of the arm in a large series of transradial approach for coronary procedures. J Interv Cardiol. 2008;21(5):380-4. 


\section{ANEXO}

Hospitais /Investigadores que colaboraram com este estudo:

- Santa Casa de Marília (Marília, SP)

André Labrunie, Wesley F. Silveira

- Hospital dos Fornecedores de Cana de Piracicaba (Piracicaba, SP)

Luiz Antonio Gubolino

- Beneficência Portuguesa de São José do Rio Preto (São José do Rio Preto, SP)

Luiz Antonio Gubolino

- Santa Casa de Franca (Franca, SP)

Ricardo Barbosa, Ulisses Gianecchini

- IMC - Instituto de Moléstias Cardiovasculares (São José do Rio Preto, SP)

Wilson M. C. Coelho

- Angiocardioimagem (Campinas, SP)

Edison Bocchi, Wilson A. Pimentel Filho, Maéve B. Correa

- Santa Casa de Piracicaba (Piracicaba, SP)

Eduardo Nicolela

- Hospital de Base de Bauru (Bauru, SP)

Caio Pessoa, Hugo A. R. Yokoyama

- Hospital Policlin (São José dos Campos, SP)

José E. Araújo, Pedro Passoni

- Hospital São Domingos (Catanduva, SP)

Fernando Stuchi Devito

- Santa Casa de Araras (Araras, SP)

Daniel Potério

- Santa Casa de Presidente Prudente (Presidente Prudente, SP)

Paulo H. Jorge, Jairon Nascimento

- Santa Casa de São José do Rio Preto (São José do Rio Preto, SP)

Mônica Buchala

- Santa Casa de Araçatuba (Araçatuba, SP)

Flavio C. Pivatelli 